\title{
An Alternative Technique in the Detection of Pancreatobiliary Malignancy: Computer-Assisted Wide Area Transepithelial Sampling
}

\author{
Samson Ferm*, Constantine Fisher, Frances Puello, Moshe Rubin, Syed Hussain and Sang Kim \\ New York Presbyterian Queens, USA
}

*Corresponding author: Samson Ferm, New York Presbyterian Queens, 56-45 Main St. Flushing New York, 11375, USA

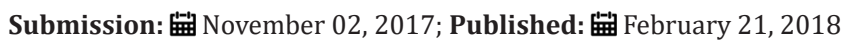

\section{Introduction}

Endoscopic Retrograde Cholangiopancreatography (ERCP) has long been used in the evaluation and treatment of biliary strictures and masses. In addition to imaging the biliary tree and pancreatic duct, ERCP allows for tissue acquisition. Both cytology and forceps biopsies can be used. Studies have shown the sensitivity of standard brush cytology to be 35-70\% [1-3] and forceps biopsy to be $40-80 \%$ [4,5]. Wide-Area Transepithelial Sampling 3Dimensional Analysis (WATS3D(R) Brush Biopsy, CDx, Suffrin, NY) computer assisted full thickness brush biopsy is a method of tissue collection that samples the total thickness of the epithelium. Improved diagnostic accuracy is thought to be due to the abrasive, rigid structure of the brush which is passed through the working port of the endoscope. Tissue acquisition is enhanced utilizing a continuous, up-and-down motion with gentle pressure that captures deeper layers that may be missed with standard forceps or brushings. Abnormal cells are then identified through a computer assisted analysis which includes 3-dimensional scanning to evaluate each portion of the full thickness specimen. When utilized to obtain biopsies of esophageal tissue, WATS3D, in conjunction with forceps biopsies, has resulted in a statistically significant increase in the number of patients diagnosed with esophageal intestinal metaplasia and dysplasia $[6,7]$. There is a paucity of literature that describes the use of WATS3D in the diagnosis of biliary malignancies. In this study, we compared the sensitivityand specificity of the WATS3D to standard brush cytology and forceps biopsy in diagnosing pancreaticobiliary malignancies at ERCP.

\section{Patients, Materials and Methods}

This was a retrospective case series design that included 40 patients (Males 18, Females 22) with a mean age of 80 (range 5596). All patients underwent ERCP to evaluate pancreaticobiliary abnormalities suspicious for malignancy found on radiographic imaging. All procedures were performed at New York Presbyterian Queens between April 2012 and December 2013. Patients underwent standard cholangiography followed by sequential traditional brushing, WATS3D cytology and forceps biopsy, or traditional brushing and WATS3D cytology only without forceps biopsy. The primary outcome measured was the detection of neoplasia with each modality. All patients underwent standard brushing during ERCP with a $2.1 \mathrm{~mm} \times 8$ French cytology brush (RX Cytology Brush, Boston Scientific; Natick, Massachusetts) over a 0.035 inch guidewire (Hyrda Jagwire ${ }^{\mathrm{TM}}$, Boston Scientific; Natick, Massachusetts). Each stricture, mass, or area of dilatation was then brushed 20 times. After brushing was completed with the standard cytology brush, WATS3D was then used. Finally, in some patients, a forceps biopsy with a $2.0 \mathrm{~mm}$ biopsy forceps (Radial Jaw $\left({ }^{\mathrm{T} M}\right)$, Boston Scientific, Natick Massachusetts) was used. All pathology was reviewed by pathologists at New York Presbyterian-Queens, NY, with final readings categorized as malignant, nonmalignant (metaplasia, hyperplasia), or atypical (dysplasia). Definitive diagnoses were obtained by forceps biopsy. Cytologic findings obtained by WATS3D and brushings were compared with the final diagnosis. A positive finding from any of the three modalities (forceps, brushing or WATS3D) was considered a positive finding and was treated as such. Atypical findings were considered positive.

Informed consent was not obtained, as the study is a retrospective case series design; all patient information was deidentified prior to data analysis. Prior to data collection, internal review board approval was obtained for chart review. All listed authors verify their full and honest academic involvement with regard to initiation of study, review of current literature regarding pancreaticobiliary malignancy detection, and collection and analysis of the data presented in this manuscript.

\section{Statistical Analysis}

Statistical analysis was performed using SPSS statistics version 21.0 (IBM corporation, Armonk, NY, USA). The simple kappa coefficient was derived using the SPSS software to obtain measure of agreement. Sensitivities and specificities were obtained using SPSS software. 


\section{Results}

In the period between April 2012 and December 2013, 40 patients with average age of 80 (range 55-96) underwent ERCP for biliary stricture as seen on radiographic imaging, with sequential biopsies procured using brushing, WATS3D and/or forceps biopsy. Of the 40 patients, 18 underwent brushing and WATS3D CDx followed by forceps biopsy and 22 patients underwent brushing and CDx without forceps biopsy. Of the 18 patients who underwent brushing, CDx and forceps biopsy, Cholangiocarcinoma was detected in 8 patients (with one result indeterminate) as diagnosed with forceps biopsy, the gold standard. The sensitivity and specificity of CDx as compared to forceps biopsy was $75 \%$ and $67 \%$, respectively. The sensitivity and specificity of cytologic brushings was $25 \%$ and 89\%, respectively (Table 1 ).

Table 1: Sensitivity and Specificity of WATS CDx and brush biopsy.

\begin{tabular}{|c|c|c|}
\hline Summary & Brush Biopsy & WATS CDx \\
\hline Sensitivity & $25 \%$ & $75 \%$ \\
\hline Specificity & $89 \%$ & $67 \%$ \\
\hline
\end{tabular}

There was moderate concordance between forceps biopsy and CDx $(k=0.414)$. Of the negative forceps biopsy results, WATS3D identified one malignancy, and 2 atypical specimen results. CDx and Brushing cytology showed moderate concordance $(k=0.4738)$, and when used in conjunction, specificity was $100 \%$. Although brushing cytology was specific, it showed only slight concordance when compared with forceps biopsy ( $k=0.1439)$. In the 22 patients who did not undergo the gold standard of forceps biopsy, WATS3D identified 3 additional malignancies, and 2 additional atypical results. WATS3D failed to identify one malignancy that was positive using forceps biopsy.

\section{Discussion}

ERCP is a technique used in the evaluation and treatment of biliary strictures and masses, in which tissue is obtained via brush and forceps biopsy. Although these modalities have a high specificity, sensitivity remains poor. Brush cytology, which is the most commonly used method for tissue sampling, has an estimated sensitivity of $35-70 \%$ when used alone and a specificity of over $90 \%$ $[4,5,8]$. In a recent study including 75 specimens, sensitivity of brush cytology was $66 \%$ [9]. The sensitivity of forceps biopsy ranges from $43-80 \%[8,10-12]$. Though the combination of brushing and biopsy has been previously evaluated, the data has been mixed. One study suggested that the combination could increase the sensitivity by 15-25\% compared to either option alone [13-15]. However, other studies suggest that the combination of brushing and biopsies were more sensitive, and the risks of doing both was not warranted $[11,13]$. This demonstrates the need for a safe, reliable, and sensitive technique for diagnosing pancreaticobiliary neoplasia.

The WATS3D system has been shown to improve diagnostic accuracy when used as an adjunct to forceps biopsy in detecting Barrett's Metaplasia [7]. In a recent randomized clinical trial, 160 patients underwent esophageal biopsy followed by WATS3D, or vice-versa. The absolute diagnostic yield increased $14.4 \%$ when
WATS3D was added to standard biopsy [16]. The use of Wide-Area Transepithelial Sampling outside of Barrett's Metaplasia has not been studied. Due to the importance of prompt surgical resection in the management of biliary malignancies, an accurate tissue diagnosis is important.

In our study, WATS3D was found to be as sensitive as standard brushing cytology and forceps biopsy in the diagnosis of biliary malignancy. Given that the reported sensitivity of forceps biopsy and brush cytology is as low as $40 \%$, the WATS3D system could double diagnostic yield when used concurrently with both modalities. Additionally, we found that WATS3D and brush cytology show moderate concordance and are moderately specific diagnostic tools. The advantages of WATS3D over traditional cytology include its stiffer brushes, which allow for deeper tissue acquisition and greater sampled surface area, as well as computer aided analysis of cells. The technical limitation to the use of WATS3D is the lack of guidewire utilization, which may limit the reach of the brush beyond certain strictures. Our study was limited, by the small sample size which most likely contributed to the poor sensitivity of brush cytology. Additionally, the sensitivity of each method might have been affected by the order of tissue acquisition, which was not analyzed is this study. Despite this, the promising results demonstrated by this study warrants further investigation. Additional studies with larger cohorts have the potential to clarify the role of Wide-Area Transepithelial Sampling brush biopsy in the diagnosis of pancreaticobiliary neoplasms.

\section{What is already known about this subject?}

I. Wide Area Transepithelial Sampling (WATS CDx) 3Dimensional brush biopsy has been used in the diagnosis of esophageal malignancy.

II. The addition of WATS CDx in conjunction with forceps biopsy has increased the diagnostic yield of esophageal neoplasia

III. WATS CDx has not been used for detection of pancreaticobiliary malignancies

\section{What are the new findings?}

V. To our knowledge, this is the first report of the use of WATS CDx brush biopsy in the diagnosis of pancreaticobiliary malignancy.

VI. WATS CDx was found to have a sensitivity of $75 \%$ and specificity of $67 \%$ in diagnosis of cholangiocarcinoma.

VII. There was moderate concordance between WATS CDx and brush cytology in diagnosis of pancreaticobiliary malignancy

VIII. How might these results change the focus of research or clinical practice?

IX. WATS CDx may add to diagnostic yield in the detection of pancreaticobiliary malignancy, however additional studies with larger cohorts will be needed to clarify the role of WATS CDx brush biopsy in this setting.

\section{References}

1. Bain VG, Abraham N, Jhangri GS, Alexander TW, Henning RC, et al. 
(2000) Prospective study of biliary strictures to determine the predictors of malignancy. Can J Gastroenterol 14(5): 397-402.

2. Adamsen S, Olsen M, Jendresen MB, Holck S, Glenthøj A (2006) Endobiliary brush biopsy: Intraand interobserver variation in cytological evaluation of brushings from bile duct strictures. Scand J Gastroenterol 41(5): 597-603.

3. Glasbrenner B, Ardan M, Boeck W, Preclik G, Möller P, et al. (1999) Prospective evaluation of brush cytology of biliary strictures during endoscopic retrograde cholangiopancreatography. Endoscopy 31(9): 712717.

4. Kurzawinski TR, Deery A, Dooley JS, Dick R, Hobbs KE, et al. (1993) A prospective study of biliary cytology in 100 patients with bile duct strictures. Hepatology 18(6): 1399-1403.

5. Schöfl R (2001) Diagnostic endoscopic retrograde cholangiopancreatography. Endoscopy 33(2): 147-157.

6. Anandasabapathy S, Sontag S, Graham DY, Frist S, Bratton J, et al. (2011) Computer Assisted Brush Biopsy Analysis for the Detection of Dysplasia in a High Risk Barrett's Esophagus Surveillance Population. Dig Dis Sci 56(3): 761-766.

7. Johanson JF, Frakes J, Eisen D EndoCDx Collaborative Group (2011) Computer Assisted Analysis of Abrasive Transepithelial Brush Biopsies Increases the Effectiveness of Esophageal Screening: A Multicenter Prospective Clinical Trial by the EndoCDx Collaborative Group. Dig Dis Sci 56(3): 767-772.

8. Trent V, Khurana KK, Pisharodi LR (1999) Diagnostic accuracy and clinical utility of endoscopic bile duct brushing in the evaluation of biliary strictures. Arch Pathol Lab Med 123(8): 712-715.

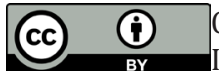

Creative Commons Attribution 4.0

International License

For possible submissions Click Here
9. Eiholm S, Thielsen P, Kromann-AH (2013) Endoscopic brush cytology from the biliary duct system is still valuable. Dan Med J 60(7): A4656.

10. Lee JG, Leung JW, Baillie J, Layfield LJ, Cotton PB (1995) Benign, dysplastic, or malignant making sense of endoscopic bile duct brush cytology: results in 149 consecutive patients. Am J Gastroenterol 90(5): 722-726.

11. Logrono R, Kurtycz DF, Molina CP, Trivedi VA, Wong JY, et al. (2000) Analysis of false negative diagnoses on endoscopic brush cytology of biliary and pancreatic duct strictures: the experience at 2 university hospitals. Arch Pathol Lab Med 124(3): 387-392.

12. Rumalla A, Baron TH, Leontovich O, Burgart LJ, Yacavone RF, et al. (2001) Improved diagnostic yield of endoscopic biliary brush cytology by digital image analysis. Mayo Clin Proc 76(1): 29-33.

13. Foutch PG (1994) Diagnosis of cancer by cytologic methods performed during ERCP. Gastrointest Endosc 40(2Pt 1): 249-252.

14. Parasher VK, Huibregtse K (1998) Endoscopic retrograde wire guided cytology of malignant biliary strictures using a novel scraping brush. Gastrointest Endosc 48(3): 288-290.

15. Fogel EL, Sherman S (1999) How to improve the accuracy of diagnosis of malignant biliary strictures. Endoscopy 31(9): 758-760.

16. Vennalaganti PR, Kaul V, Wang KK, Falk GW, Shaheen NJ, et al. (2017) Increased Detection of Barrett's Esophagus-associated Neoplasia Using Wide-Area Transepithelial Sampling: A Multicenter, Prospective, Randomized Trial. Gastrointest Endosc 87(2): 348-355.

\section{Your subsequent submission with Crimson Publishers will attain the below benefits}

- High-level peer review and editorial services

- Freely accessible online immediately upon publication

- Authors retain the copyright to their work

- Licensing it under a Creative Commons license

- Visibility through different online platforms

- Global attainment for your research

- Article availability in different formats (Pdf, E-pub, Full Text)

- Endless customer service

- Reasonable Membership services

- Reprints availability upon request

- One step article tracking system 